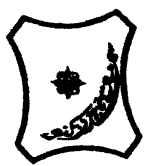

\title{
SUB-ACUTE INSULIN THERAPY DOES NOT AFFECT LONG-TERM VISIO- SPATIAL LEARNING AND MEMORY IN MICE ASSESSED USING BARNES MAZE
}

\author{
${ }^{* 1}$ Yarube, I.U., ${ }^{2}$ Ayo, J.O., ${ }^{2}$ Magaji, R.A. ${ }^{4}$ Umar, I.A., ${ }^{3}$ Alhassan, A.W., and ${ }^{3}$ Saleh, M.I.A. \\ ${ }^{1}$ Department of Physiology, Faculty of Basic Medical Sciences, College of Health Sciences, Bayero University, \\ Kano-Nigeria. \\ ${ }^{2}$ Department of Veterinary Physiology, Faculty of Veterinary Medicine, Ahmadu Bello University, Zaria-Nigeria. \\ ${ }^{3}$ Department of Human Physiology, Faculty of Medicine, Ahmadu Bello University, Zaria-Nigeria. \\ ${ }^{4}$ Department of Biochemistry, Faculty of Science, Ahmadu Bello University, Zaria-Nigeria. \\ ${ }^{*}$ Correspondence: dryarube@yahoo.com
}

\begin{abstract}
Insulin is a common hypoglycaemic agent used to treat diabetes, but it has also been reported to exert other effects on the body including modulation cognition. Reported findings on insulin effect on learning and memory are scanty and often conflicting. This study was aimed at evaluating the effect of sub-acute insulin therapy on visio-spatial learning and memory using Barnes maze. Twelve young mice of both sexes, weighing between $20-22 \mathrm{~g}$, were divided into control and insulin-treated groups $(n=6)$. They were administered subcutaneously with deionized water (control) or insulin (10 I.U./kg/day) for seven days. During the last three days of treatments, the mice were subjected to two-day training and one-day probe trial of Barnes maze. Number of primary head searches on day 2 was reduced compared to day 1 for both the insulin-treated (4.17 $\pm 0.8 \mathrm{~s}$ and $11.45 \pm 1.9 \mathrm{~s})$ and control $(10.0 \pm 3.2 \mathrm{~s}$ and $19.95 \pm 4.5 \mathrm{~s})$ groups $(P<0.05)$, but the values obtained in the two groups did not differ $(P>0.05)$. Similarly, there was no difference between the insulin-treated and control groups in latency to locate the escape hole, time spent and number of head searches per quadrant.

It was concluded that sub-acute insulin therapy did not affect long-term visio-spatial learning and memory in mice.
\end{abstract}

Keywords: insulin, visio-spatial learning and memory, long-term memory, Barnes maze, mice

\section{INTRODUCTION}

Learning was described as the act of acquiring new, or modifying and reinforcing existing knowledge, behaviors, skills, values, or preferences and may involve synthesizing different types of information (Daniel et al. 2011). Memory is the storage and retrieval of information (Inkster and Frier, 2012). Visio-spatial cognition is concerned with the acquisition, organization, utilization, and revision of knowledge about spatial environments using visual cues. Visio-spatial memory can take the form of working, short-term or long-term memory. A person's visio-spatial memory is required in order to navigate around a familiar location, just as a rodent's visiospatial memory is needed to learn the location of food or platform at the end of a maze (Denis and Loomis, 2007).Barnes maze test was first developed by Carol Barnes in 1979 (Barnes, 1979). The test was originally developed for use in rats to overcome stress induced by swimming in the Morris water maze, but was later adapted for mice. The test relies on the innate tendency of mice to escape from an open and bright space into closed and darker space (Aida et al., 2013).

Insulin - a hypoglycaemic agent used in the management of diabetes - is a common drug used in
sub-Sahara Africa and the world at large. There is high prevalence of diabetes in the region and globally, translating to about 387 million people in 2014; and an estimated 592 million persons will live with the disease by 2035 (Chinenye and Young, 2011; IDF, 2014). Apart from its hypoglycaemic effects, insulin has other effects on the body including modulation of cognition (Ghasemiet al., 2013). However, the reported effects of insulin on visio-spatial learning and memory are scanty and often conflicting. For example, while McNayet al. (2010) reported improvement, Kamal et al. (2013) found impairment of hippocampal memory process caused by insulin.

The aim of this study was to determine the effect of sub-acute insulin therapy on long-term visio-spatial learning and memory using Barnes maze.

\section{MATERIALS AND METHODS}

Animals and Care

Young mice of both sexes (obtained from Department of Pharmacology, Faculty of Pharmacy, Ahmadu Bello University, Zaria), weighing between $20-22 \mathrm{~g}$, were used for the study. They were kept in large cages and given access to feed and drinking water ad libitum during acclimatization and throughout the experimental period. 
They were maintained under the prevailing natural light-dark cycle with photophase between 6:19 18:42 hours. Experimental protocols were approved by Institutional Research Committee and were in accordance with the guidelines for animal research, as stated in the Guide for the Care and Use of Laboratory Animals (National Academy of Sciences, 2011).

\section{Grouping and treatments}

Twelve mice were divided into control and insulintreated groups ( $n=6$ per group). They were treated withdeionized water (control) or insulin (Actrapid, Novo Nordisk A/S, Denmark) at 10 I.U./kg/day, subcutaneously using insulin syringe daily between the hours of 8:00 - 9:00 am (Sharma et al., 2007). Behavioural tests were done 30 minutes after the last insulin injection, following one week of insulin administration (Francis et al., 2008).

Assessment of long-term visio-spatial learning and memory using Barnes maze

In the present study, the Barnes maze paradigm consisted of the habituation phase (1 day), training phase ( 2 days) and probe trial ( 1 day). Each mouse had only one trial during the habituation phase (first day) of this paradigm. During this phase, a mouse was introduced into the centre of the maze under an opaque chamber and was allowed to stay there for 30 s. The chamber was lifted, the overhead bright light (aversive stimulus/ negative reinforcement) was put on, and the mouse was guided to the target hole (leading to the escape box) within 10-15 s. Once the mouse had located the target hole it was allowed to independently enter the escape box within 3 minutes. If it failed to enter, it was gently taken into it and allowed to stay for $1 \mathrm{~min}$. No parameters were recorded during this phase.

During the training phase, a mouse was positioned in an opaque chamber in the centre of the maze and allowed to stay for $15 \mathrm{~s}$. The chamber was then lifted, the overhead bright light was put on, and the mouse was allowed 2 min to explore the maze and locate the target whole. If it located the target hole and entered into the escape box within this period, it was allowed to stay there for $1 \mathrm{~min}$. If it failed to locate the target hole at the expiration of the $2 \mathrm{~min}$, it was gently guided to the target hole and allowed 3 min to enter into the escape box independently. If the mouse failed to enter after this period, it was then gently taken into the escape box and allowed there for 1 min. Furthermore, if a mouse failed to enter into the escape box independently after locating the target hole, it was allowed 3 min to do so. If it failed, it was gently introduced into the escape box and allowed 1 min to stay. Three and two trials per day were conducted for each mouse on day 1 and day 2 of the training phase, respectively. An overhead video camera recorded the activities of the mice for subsequent quantification. Primary head searches (number of head dips into any holes that did not have the escape box before locating the escape hole) and latency (time taken) to locate the escape box (primary latency), were recorded. If a mouse failed to enter into the hole after locating it for the first time, recordings were stopped (primary latency and primary head search), but the mouse was allowed to continue exploring the maze till the expiration of the 2-minute period.

During the probe trial (on the $4^{\text {th }}$ day of the experiment), the mice were tested for remembering what had been previously learned. Each mouse was positioned in the centre chamber for $15 \mathrm{~min}$. The chamber was lifted, the overhead bright light was put on, and the mouse was given 2 min to explore the maze. Time spent per quadrant and number of head searches per quadrant was recorded. After each trial, the maze was wiped with a cloth dipped in $70 \%$ ethanol and allowed to dry to remove any olfactory cue.

\section{Statistical analyses}

Data were collated and analyzed using Statistical Package for Social Sciences(SPSS) version 20.0 software (SPSS Inc., Chicago, Il., U.S.A.), and expressed as mean \pm S.E.M. General linear model repeated measure ANOVA was used to compare means. Values of $\mathrm{P}<0.05$ were considered significant.

\section{RESULTS AND DISCUSSION}

Number of primary head searches and latency (time taken) to locate escape hole

The difference in numbers of primary head searches between days 1 and 2 for both the insulin-treated (11.45 $\pm 1.9 \mathrm{~s}$ and $4.17 \pm 0.8 \mathrm{~s})$ and control (19.95 \pm $4.5 \mathrm{~s}$ and $10.0 \pm 3.2 \mathrm{~s}$ ) groups werehighly significant, (Pillai's Trace $=0.623, F_{(1,10)}=16.527, P=0.002$, $\mathrm{Eta}^{2}=0.623$ ) (Figure 1). This indicates that the mice had learned the position of the escape hole and were able to find it with fewer searches/errors. However, this parameter was similar for the two groups $\left(F_{(1,}\right.$ 10) $\left.=4.133, P=0.069, \mathrm{Eta}^{2}=0.292, \mathrm{n}=6\right)$, suggesting that insulin did not affect (impair or improve) the mice ability to learn the position of the escape hole.

There was no significant difference between latencies recorded on days 1 and 2 for the insulin-treated $(113.0 \pm 4.5 \mathrm{~s}$ and $97.19 \pm 10.5 \mathrm{~s})$ and control (98.95 $\pm 2.5 \mathrm{~s}$ and $92.17 \pm 18.5 \mathrm{~s}$ ) groups (Wilks' lambda $=$ $0.883, F_{(1,10)}=1.319, P=0.278$, multivariate partial Eta squared $\left[\mathrm{Eta}^{2}\right]=0.117, \mathrm{n}=6$ ) (Figure 2). Latencies obtained for the two groups also did not differ $\left(F_{(1,10)}=0.638, P=0.443, \mathrm{Eta}^{2}=0.060, \mathrm{n}=\right.$ $6)$. This finding indicates that insulin did not affect learning in the treated mice.

During the 2-day training phase, the animals have learned the location of the escape hole as indicated by reduced number of head searches and/or fewer committed errors in trying to locate the escape hole. However, the time taken (latency) to locate the hole remained the same, rather than reduce for both the groups. This finding may be related to the less stressful nature of Barnes maze, compared to Morris water maze as suggested by Harrison et al. (2009); occasioned by weaker aversive stimuli (bright light compared to deep water of Morris water maze), hence less motivation to escape and absence of reduction in day 2 latency. 


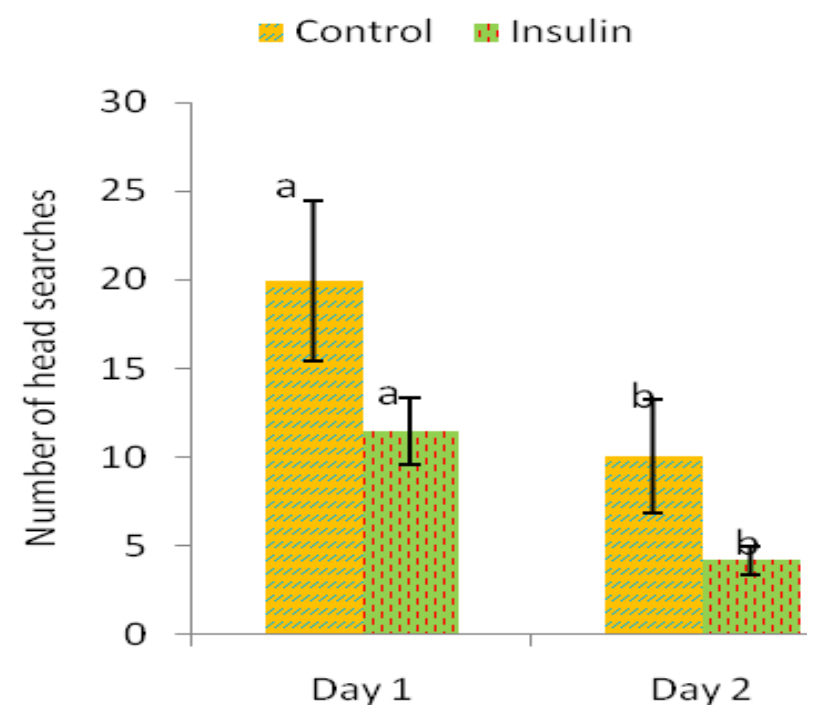

Figure 1: Number of primary head searches by control and insulin-treatedmice during a 2-day training of Barnes maze task. ${ }^{a, b}=$ Columns with different superscript lettersare significantly $(P<0.05)$ different. (Mean \pm S.E.M, $n=$ 6)

\section{Control Insulin}

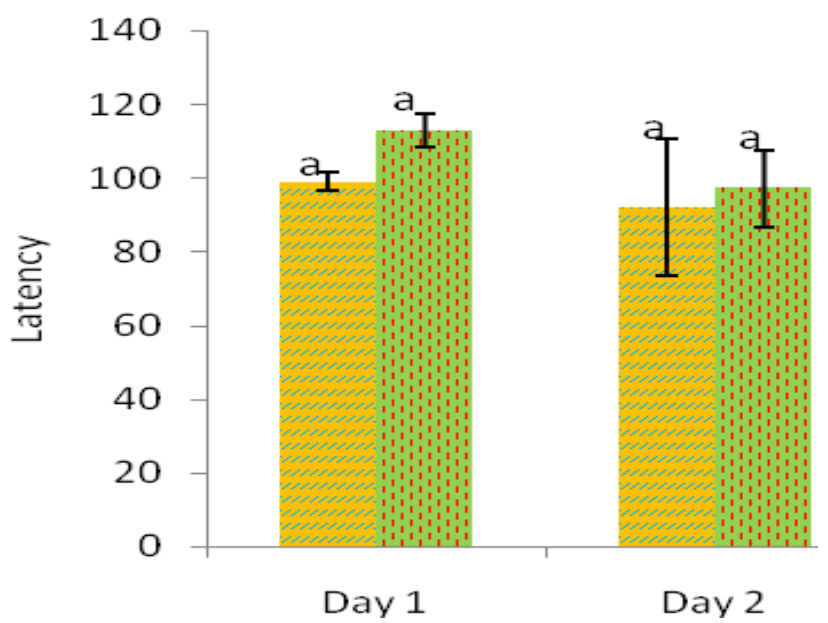

Figure 2: Latency (seconds) to locate escape hole by control and insulin-treated mice during a 2-day training of Barnes maze task. ${ }^{a}=$ Columns with the same superscript letters are the same $(P>0.05)$. (Mean \pm S.E.M, $\mathrm{n}=$ 6)

Time spentper quadrant and number of head searches per quadrant

The mice in the insulin-treated groups spent $19.67 \pm$ $7.0 \mathrm{~s}, 20.33 \pm 4.3 \mathrm{~s}, 15.67 \pm 5.1 \mathrm{~s}$ and $24.67 \pm 9.1 \mathrm{~s}$ in quadrants $1,2,3$ and 4 , respectively. The control animals spent $19.33 \pm 15.0 \mathrm{~s}, 18.00 \pm 7.0 \mathrm{~s}, 28.00 \pm$ $7.0 \mathrm{~s}$ and $19.33 \pm 6.8 \mathrm{~s}$, in quadrants $1,2,3$ and 4, respectively (Figure 3 ).There was no significant difference between the groups in time spent byanimals per quadrant (Wilks' Lambda $=0.962, F_{(3,}$ 20) $\left.=0.105, \quad P=0.955, \mathrm{Eta}^{2}=0.038, \mathrm{n}=6\right)$, indicating no preference of the target quadrant by both the groups.

Number of head searches was $1.58 \pm 1.33,1.33 \pm$ $0.6,2.17 \pm 1.4$ and $1.0 \pm 0.8$ in quadrants $1,2,3$, and 4 , respectively, for the insulin-treated group; and the values were $2.17 \pm 1.4,2.83 \pm 1.0,4.00 \pm 1.0$ and $2.50 \pm 1.0$, respectively, in quadrants $1,2,3$ and 4 for the control group (Figure 4). The difference in the number of head searches per quadrant between the two groups was insignificant (Wilks' Lambda = $0.662, F_{(3,20)}=1.362, P=0.332, \mathrm{Eta}^{2}=0.038, \mathrm{n}=$ $6)$, indicating no preference of the target quadrant by both groups.

During the probe trial, the performance of the insulintreated and control animals was the same as the mice showed no preference of the target quadrant, measured by the amount of time spent and number of head searches per quadrant. Overall, the insulintreated mice had the same performnce in all the four parameters, compared to the vehicle-treated controls. The result suggests that there was no effect of insulin treatment on learning and memory in the mice. It was previously reported that insulin signalling is involved in the physiology of memory in man and lower animals (Lin et al., 2010; Liu et al., 2013; De Felice et al., 2014;Chambers et al., 2015). Others reported impairment of memory due to insulin (Kamal et al., 2012; Krikorian, 2013). 
The result of this study, though contrary to the above findings agree with the finding of Backeström et al. (2015), who reported that plasma insulin levels and insulin resistance were not associated with episodic or semantic memory in women or in men after adjustments for many factors, including age, fasting glucose, 2-hour glucose, BMI, education, smoking, cardiovascular disease, hypertension, cholesterol, and physical activity. The reason for the differences in findings may be due to inherent tendency for most physiological processes to vary based on many influencing circumstances such as duration and quantity of exposure to the influencing factors.

Control Insulin

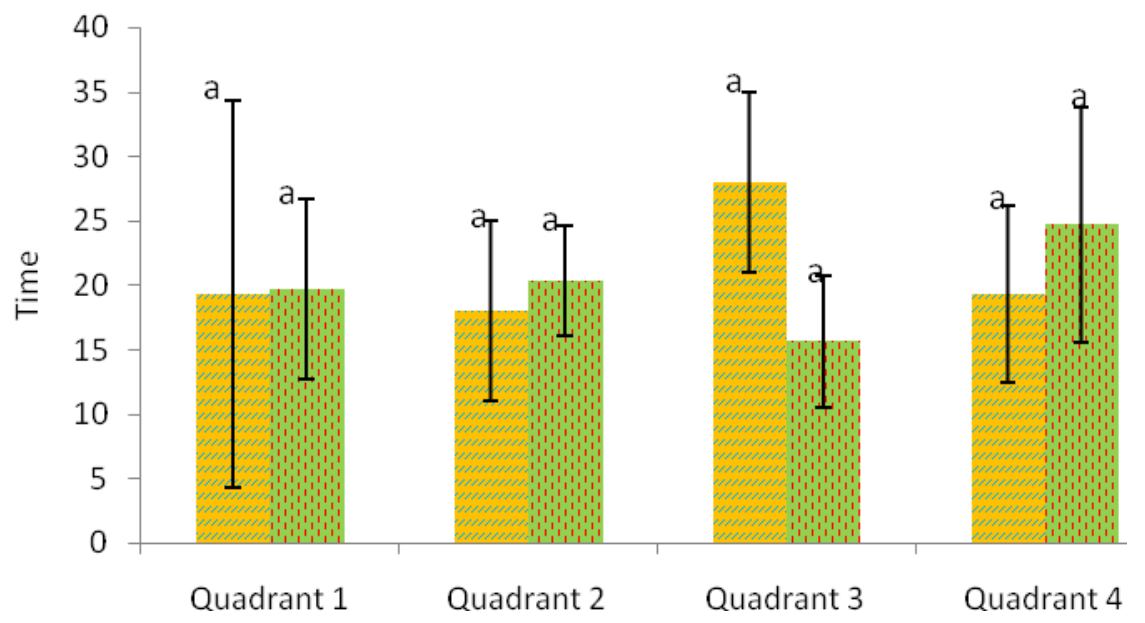

Figure 3: Time (seconds) spent per quadrant by control and insulin-treated mice during a 1-day probe trial of Barnes maze task. ${ }^{a}=$ Columns with the same superscript letters are the same $(P>0.05)$. (Mean \pm S.E.M, $\left.n=6\right)$

$$
\text { Control } \text { Insulin }
$$

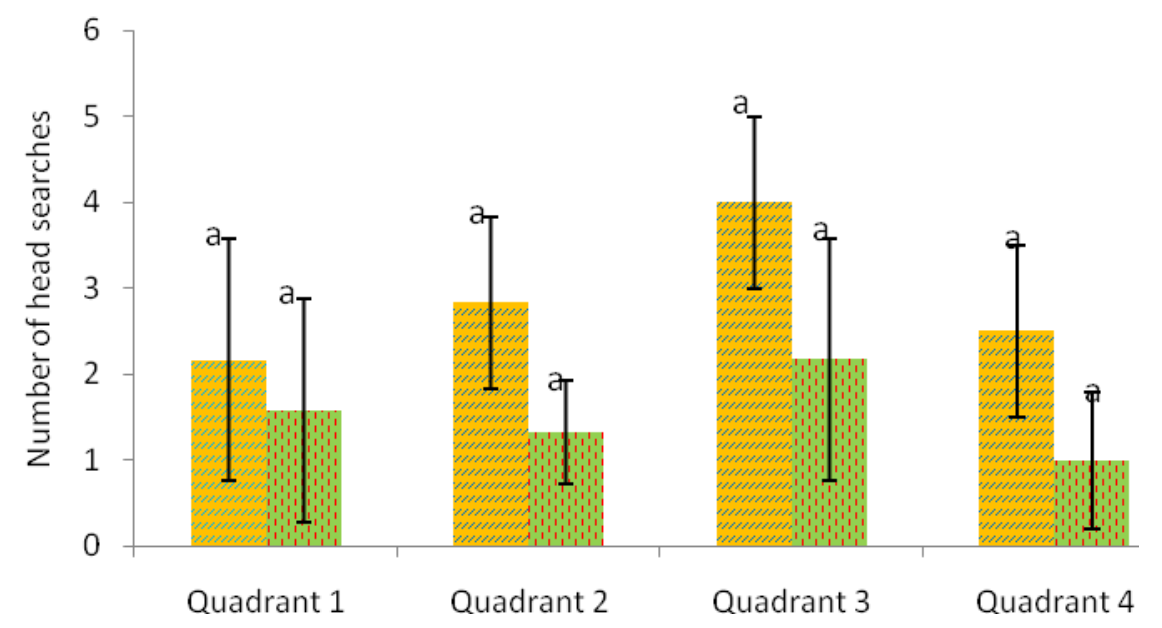

Figure 4: Number of head searches per quadrant for control and insulin-treated mice during a 1- day probe trial of Barnes maze task. ${ }^{a}=$ Columns with the same superscript letters are the same $(P>0.05)$. (Mean \pm S.E.M, n = 6)

\section{CONCLUSION}

It was concluded that sub-acute insulin therapy did not affect long-term visio-spatial learning and memory in the treated mice. At this relatively early stage, further investigations are required to accumulate sufficient data to fully ellucidate the effects of insulin on the different types of learning and memory in varied circumstances.

\section{Conflict of Interest}

Authors declare that there is no conflict of interest regarding this publication.

\section{Acknowledement}

The authors wish to thank Bayero University, Kano, and Tertiary Education Trust Fund (TETFund) for funding this work. 


\section{Contribution of authors}

Yarube conceived the idea, collected data and participated in drafting and reviewing the manuscript. Ayo guided the work, participated in data collection/analysis, drafting and reviewing the

\section{REFERENCES}

Aida, A., Tingyu, L., and Gal, B. (2013). A shortened Barnes maze protocol reveals memory deficits at 4-months of age in the tripletransgenic mouse model of Alzheimer's disease. PloS One, 8(11): 80355, DOI:10.1371/journal.pone.0080355

Backeström, A., Eriksson, S., Nilsson, L., Olsson, T. and Rolandsson, O. (2015). Glucose but not insulin or insulin resistance is associated with memory performance in middle-aged nondiabetic women: a cross sectional study. Diabetology and Metabolic Syndrome, 7: 20 28.

Barnes, C.A. (1979). Memory deficits associated with senescence: a neurophysiological and behavioral study in the rat. Journal of Comparative and Physiological Psychology, 93(1): 74 - 104.

Chambers, D.B., Androschuk, A., Rosenfelt, C., Langer, S., Harding, M. and Bolduc, F.V. (2015). Insulin signaling is acutely required for long-term memory in Drosophila. Frontiers in Neural Circuits, 9: 8 - 12.

Chinenye S, Young E. (2011). State of Diabetes Care in Nigeria: A Review. The Nigerian HealthJournal 11(4):101-106.

Daniel, L.S., Daniel T.G., and Daniel, M.W. (2011).Memory. In: Psychology, 2nd Edition, Worth Publishers, New York,pp. 264-271.

De Felice, F.G.D., Lourenco, M.V., Ferreira, S.T. (2014). How does brain insulin resistance develop in Alzheimer's disease? Alzheimer's and Dementia, 10: S26 - S32.

Denis, M. and Loomis, J. (2007). Perspectives on human spatial cognition: memory, navigation, and environmental learning. Psychological Research, 71(3): 235 - 239.

Francis, G.J., Martinez, J.A., Liu, W.Q., Xu, K., Ayer, A., Fine, J., Tuor, U.I., Glazner, G., Hanson, L.R., Frey II, W.H. and Toth, C.C. (2008). Intranasal insulin prevents cognitive decline, cerebral atrophy and white matter changes in murine type I diabetic encephalopathy. Brain, 131: 3311 - 3334.

Ghasemi, R, Haeri, A., Dargahi, L., Mohamed, Z. and Ahmadiani, A. (2013). Insulin in the brain: sources, localization and functions. Molecular Neurobiology, 47(1): 145 - 71.

Harrison, F.E., Hosseini, A.H. and McDonald, M.P. (2009). Endogenous anxiety and stress responses in water maze and Barnes maze spatial memory tasks. Behavioural Brain Research. 198(1): 247 - 251.

Inkster, B. and Frier, B.M. (2012).The effects of acute hypoglycaemia on cognitive function in type 1 diabetes. British Journal of Diabetes and Vascular Disease, 12(5): 221-226. manuscript. Magaji and Umar participated in data collection/analysis, drafting and reviewing the manuscript. Alhassan and Saleh participated in data interpretation, drafting and reviewing the manuscript.

International Diabetes Federation (IDF).IDF Diabetes Atlas 2014, $6^{\text {th }}$ Edition.https://www.idf.org/sites/default/files /Atlas-poster-2014_EN.pdf (accessed on 28th Jan., 2016).

Kamal, A., Ramakers, G.M.J., Gispen, W.H. and Biessels, G.J. (2012).Effect of chronic intracerebroventricular insulin administration in rats on the peripheral glucose metabolism and synaptic plasticity of CA1 hippocampal neurons. Brain Research, 1435: 99 - 104.

Kamal, A., Ramakers, G.M.J., Gispen, W.H., Biessels, G.J., and Al Ansari, A. (2013).Hyperinsulinemia in rats causes impairment of spatial memory and learning with defects in hippocampal synaptic plasticity by involvement of postsynaptic mechanisms. Experimental Brain Research, 226: $45-51$.

Krikorian, R. (2013). Metabolic disturbance and dementia: A modifiable link. Current Psychiatry, 12 (3): 17 - 23.

Lin, C.H.A., Tomioka, M., Pereira, S., Sellings, L., Iino, Y., and van der Kooy, D. (2010).Insulin signaling plays a dual role in Caenorhabditiselegansmemory acquisition and memory retrieval. The Journal of Neuroscience, 30(23): 8001 - 8011.

Liu, P., Zou, L., Jiao, Q., Chi, T., Ji, X., Qi, Y., Xu, Q. andWang, L. (2013). Xanthoceraside attenuates learning and memory deficits via improving insulin signaling in STZ-induced AD rats.Neuroscience Letters, 543: 115 120.

McNay, E.C., Ong, C.T., McCrimmon, R.J., Cresswell, J., Jonathan, S. Bogan, J.S. and Sherwin, R.S. (2010). Hippocampal memory processes are modulated by insulin and high-fatinduced insulin resistance. Neurobiology of Learning and Memory, 93(4): 546 - 553.

National Academy of Sciences (2011).Guide for the Care and Use of Laboratory Animals. National Academies Press, Washington, D.C., U.S.A., 246 pp.

Sharma, S., Chopra, K. and Kulkarni, S.K. (2007). Effect of insulin and its combination with resveratrol or curcumin in attenuation of diabetic neuropathic pain: participation of nitric oxide and TNF-alpha. Phytotherapy Research, 21(3): 278 - 283. 